ORIGINAL ARTICLE

\title{
Hydrofluoroalkane-beclomethasone versus chlorofluorocarbon-beclomethasone delivery in neonatal models
}

\section{H Cole, J P Mitchell, M P Foley, M W Nagel}

\author{
See end of article for \\ authors' affiliations \\ ..................... \\ Correspondence to: \\ Dr Cole, Department of \\ Neonatology, Beth Israel \\ Deaconess Medical \\ Center, 330 Brookline \\ Ave, Boston, MA 02115, \\ USA; ccole@ \\ bidmc.harvard.edu \\ Accepted \\ 7 November 2003
}

Arch Dis Child Fetal Neonatal Ed 2004;89:F417-F418. doi: 10.1136/adc.2002.022905

\begin{abstract}
Dose delivery of hydrofluoroalkane-beclomethasone and chlorofluorocarbon-beclomethasone was compared during in vitro neonatal simulations: mechanical ventilation with $40 \%$ and $100 \%$ relative humidity + Neonatal Chamber-Ventilator System/endotracheal tube; manual ventilation + Neonatal Chamber/endotracheal tube; "spontaneous breathing" + Neonatal Chamber/face mask without/with manual assistance. The delivery of hydrofluoroalkane-beclomethasone was significantly greater in each simulation.
\end{abstract}

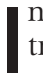
nhaled glucocorticoid therapy for the prevention or treatment of bronchopulmonary dysplasia has shown either modest or no improvements in pulmonary outcomes. ${ }^{12}$ The limited response may result from inadequate, inconsistent delivery of glucocorticoids to neonatal lungs. The production of finer aerosol particle size in a hydrofluoroalkane (HFA) reformulation of inhaled glucocorticoids and new designs of holding chambers may improve neonatal aerosol delivery. ${ }^{3}$ In vitro studies of aerosol particle size reported that the mass median aerodynamic diameter of HFA-beclomethasone dipropionate QVAR (HFA-BDP) from the actuator mouthpiece was $1.1 \mu \mathrm{m}$ in contrast with $3.4 \mu \mathrm{m}$ for chlorofluorocarbon-beclomethasone dipropionate (CFC-BDP). ${ }^{4}$

There are no published in vitro data comparing aerosol delivery of HFA-BDP and CFC-BDP using neonatal simulations. One cannot apply findings from studies in adults to the treatment of neonates. It is necessary to examine unique aerosol-device interactions and delivery technique related factors applicable to neonates. ${ }^{5}$ The objective of this study was to compare the dose delivery of HFA-BDP and CFC-BDP exiting either an endotracheal tube or face mask during five in vitro neonatal simulations. We tested the hypothesis that more HFA-BDP than CFC-BDP is delivered in each simulation. A more detailed version can be found at http:// adc.bmjjournals.com/supplemental/.

\section{METHODS}

We compared two glucocorticoid formulations (HFA-BDP (QVAR50; 3M Pharmaceuticals, London, ON, Canada; $50 \mu \mathrm{g}$ / dose) and CFC-BDP (Vanceril50; Schering, Pointe Claire, Quebec, Canada Inc; $50 \mu \mathrm{g} /$ dose) ) and used two prototype aerosol delivery chambers (Neonatal Chamber-Ventilator System and Neonatal Chamber; Trudell Medical International, London, ON, Canada).

The first two simulations assessed delivery using the Neonatal Chamber-Ventilator System and a $2.5 \mathrm{~mm}$ endotracheal tube during mechanical ventilation at $40 \%$ and $100 \%$ relative humidity. The third simulation assessed delivery using the Neonatal Chamber and endotracheal tube during manual ventilation. The fourth and fifth scenarios simulated delivery to spontaneously breathing neonates using the
Neonatal Chamber attached to a face mask without and with manual ventilation using a flow inflating resuscitation bag.

Each simulation used ventilation parameters similar to those used with neonates: rate $=40$ cycles/minute; tidal volume $=8-11.6 \mathrm{ml}$; positive end expiratory pressure $=$ $5 \mathrm{~cm} \mathrm{H}_{2} \mathrm{O}$; peak inspiratory pressure $=20 \mathrm{~cm} \mathrm{H}_{2} \mathrm{O}$; peak inspiratory flow rate $=9$ litres/min; inspiratory/expiratory (I/E) ratio = $1: 4-1: 4.8$.

Each inhaler was shaken before actuation. Actuation occurred at the beginning of the inspiratory cycle and was followed by six breathing cycles. Three actuations of each drug were delivered at 45 second intervals for each measurement. Total emitted dose per actuation (TED, $\mu \mathrm{g}$ ) was based on these measurements, divided by the number of actuations delivered from the metered dose inhaler. Delivery efficiency (\%) was calculated on a percentage basis (TED divided by label claim dose ex-metering valve $\times 100$ ). Beclomethasone concentrations were determined by high performance liquid chromatography/ultraviolet spectrophotometry.

Comparisons of TED and delivery efficiency were made among systems for a single drug (analysis of variance) and between drug formulations within a single system ( $t$ test). Differences were deemed significant when $\mathrm{p}<0.05$.

\section{RESULTS}

Table 1 shows TED and delivery efficiency for each simulation. More HFA-BDP was delivered than CFC-BDP. Manual ventilation enhanced and humidification reduced aerosol delivery.

\section{DISCUSSION}

This study illustrates important differences in aerosol delivery between HFA-BDP and CFC-BDP formulations and differences among techniques used in neonatal care. The finding of more effective delivery of HFA-BDP than CFC-BDP is not surprising given its finer particle size.

Abbreviations: CFC-BDP, chlorofluorocarbon-beclomethasone dipropionate; HFA-BDP, hydrofluoroalkane-beclomethasone dipropionate 
Table 1 Neonatal simulations of HFA-BDP and CFC-BDP delivery through an endotracheal tube using the Neonatal Chamber-Ventilator System or the Neonatal Chamber (simulations 1-3) and using the Neonatal Chamber and face mask without and with manual ventilation assistance (simulations 4 and 5)

\begin{tabular}{llllll}
\hline Formulation & Simulation 1 & Simulation 2 & Simulation 3 & Simulation 4 & Simulation 5 \\
\hline HFA-BDP* & $4.4(0.7)(8.8 \%)$ & $1.5(0.2)(3.0 \%)$ & $6.5(0.6)(13.0 \%)$ & $4.1(1.6)(8.2 \%)$ & $26.6(3.1)$ \\
CFC-BDP** & $0.4(0.3)(0.8 \%)$ & $0.02(0.08)(0.04 \%)$ & $1.3(0.5)(2.6 \%)$ & $2.3(0.7)(4.6 \%)$ & $21.6(4.3)$ \\
p Value & $<0.0001$ & $<0.0001$ & $<0.0001$ & 0.0007 & $(43.2 \%)$ \\
\hline
\end{tabular}

Dose delivered is expressed as mean (SD) total emitted dose (TED) ( $\mu \mathrm{g})$ and efficiency (TED/label claim dose) (\%). Three actuations/measurement; 5 measurements per holding chamber $\times 3$ holding chambers per simulation $=15$ samples per simulation. Simulation 1, Mechanical ventilation, $40 \%$ relative humidity (NCVS/ET); simulation 2, mechanical ventilation, 100\% relative humidity (NCVS/ET); simulation 3, manual ventilation (NC/ET); simulation 4 , no manual ventilation assistance ( $\mathrm{NC} /$ face mask); simulation 5 , manual ventilation assistance (NC/face mask) *Simulation 1 and 2 v $3, \mathrm{p}<0.001 ; 4 \vee 5, \mathrm{p}<0.001$.

**Simulation $1 \vee 3, p=0.003 ; 2$ v 3, p<0.001; 4 v 5, $\mathrm{p}<0.001$.

HFA-BDP, Hydrofluoroalkane-beclomethasone; CFC-BDP, chlorofluorocarbon-beclomethasone; ET, endotracheal tube; NCVS, Neonatal Chamber-Ventilator System; NC, Neonatal Chamber.

Awareness that humidification increases aerosol particle size $^{5}$ and reduces aerosol delivery is clinically relevant because gas supply through ventilation circuits is normally maintained close to $100 \%$ relative humidity to avoid drying of airway mucosa. ${ }^{6}$

Manual ventilation enhanced aerosol delivery through an endotracheal tube and face mask even though mechanical and spontaneous breaths used similar ventilation parameters. Greater aerosol delivery by manual ventilation may be influenced by the lower relative humidity and differences in flow dynamics. Use of manual ventilation requires close attention to the technique and consistency with which it is applied to minimise variability in aerosol delivery.

Neonatal aerosol therapy is maximised by using formulations with delivery devices that optimise aerosol particle size and dose delivery in conjunction with consistent good technique of aerosol administration. Neonatal ChamberVentilator System and Neonatal Chamber devices are designed to minimise loss of physiologically important fine particles $<3.1 \mu \mathrm{m}$ and by minimising "dead volume" where exhaled breath can dilute the aerosol.

In vitro studies provide important information on distribution of aerosol particle size and estimates of aerosol delivery with specific "medication formulation/device/technique" combinations. It is important to recognise the limitations of in vitro models. In vitro delivery may overestimate the dose ultimately deposited in the lungs as it represents any aerosol that exited the endotracheal tube or face mask and deposited on the filters, including retention of submicron particles $(<1.1 \mu \mathrm{m})$ by the filters. In vivo deposition will be attenuated by deposition processes that take place in the nares, mouth, oropharynx, and upper and conducting airways plus swallowing of aerosol particles and exhalation of some submicron particles.

This in vitro study may influence the design of in vivo deposition studies, dose-response studies, and may ultimately affect the technique used to evaluate the efficacy and safety of HFA-glucocorticoid treatment in neonatal clinical trials. The availability of extra-fine HFA-glucocorticoid aerosol offers new opportunities to assess potential clinical efficacy and safety of this medication in the treatment of evolving or established bronchopulmonary dysplasia.

\section{ACKNOWLEDGEMENTS}

We gratefully acknowledge Cathy C Doyle, BSC and Sara L Bates for their technical assistance in conducting the simulations and measurements, Drs John Fiascone and James Hagadorn for their critical reviews of the manuscript, and Dr Christopher Schmid, Stacy Supran, and Lori Lyn Price for their assistance in the statistical analysis and review of the manuscript.

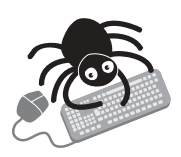

A more detailed version of this study can be found at http://adc.bmijournals.com/supplemental/.

\section{Authors' affiliations}

C H Cole, The Floating Hospital for Children, Tufts-New England Medical Center, Tufts University School of Medicine, Boston, MA, 021 11, USA J P Mitchell, M P Foley, M W Nagel, Trudell Medical International, London, Ontario, Canada, N5V 5G4

Disclosure: The manufacturer of chlorofluorocarbon-beclomethasone dipropionate (CFC-BDP; $50 \mu \mathrm{g} /$ dose; Vanceril50) is Schering, Canada Inc. The manufacturer of hydrofluoroalkane-beclomethasone dipropionate (HFA-BDP; $50 \mu \mathrm{g} /$ dose; QVAR50) is $3 \mathrm{M}$ Pharmaceuticals (Canada). The manufacturer of the two holding chambers (Neonatal Chamber-Ventilator System and Neonatal Chamber) is Trudell Medical International, London, ON, Canada. Trudell Medical International employs all co-authors, except for CHC. In vitro assays were conducted in the medical aerosol laboratory of Trudell Medical International. CHC has no financial arrangements and no conflict of interest with Trudell Medical International, Schering, Canada, or $3 \mathrm{M}$ Pharmaceuticals (Canada). CHC and biostatisticians of Tufts University, School of Medicine analysed the data objectively and free of bias by collaborators and co-authors of Trudell Medical International. Trudell Medical International provided financial support for this investigator initiated research.

\section{REFERENCES}

1 Cole CH, Colton T, Shah BL, et al. Early inhaled glucocorticoid therapy to prevent bronchopulmonary dysplasia. N Engl J Med 1999:340:1005-10.

2 Fok T, Lam K, Dolovich M, et al. Randomized controlled study of early use of inhaled corticosteroid in preterm infants with respiratory distress syndrome. Arch Dis Child Fetal Neonatal Ed 1999;80:F203-8.

3 Dolovich M. New delivery systems and propellants. Can Respir J 1999;6:290-5.

4 Leach C. Enhanced drug delivery through reformulating MDIs with HFA propellants: drug deposition and its effect on preclinical and clinical programs. In: Dalby RN, Byron PR, Farr SJ, eds. Respiratory drug delivery V. Buffalo Grove, IL: Interpharm Press, 1996:133-44.

5 Cole CH. The use of aerosolized medicine in neonates. In: Frantz, ID III, eds. Neonatal respiratory disease. Newtown, PA: Associates in Medical Marketing Co, 2000;10(4):1-12.

6 Finlay WH. The mechanics of inhaled pharmaceutical aerosols. London: Academic Press, 2001:47-91. 